\title{
Cell Fractionation and Protein Extraction from Activated Sludge by Motion Control of Ferrite Particles
}

\author{
Masaya Endo ${ }^{* a)}$ Member, Mahmudul Kabir ${ }^{*}$ Member \\ Noboru Yoshimura** $^{* *}$ Life Member
}

(Manuscript received March 1, 2018, revised Aug. 18, 2018)

\begin{abstract}
Recently, research on gene for different biological substances has attracted significant attention. Protein extraction is the first step to get the information about DNA, RNA, etc. Protein is also essential in manufacturing medicine, food and various types of biological reagents. In order to extract protein from the cell of a biological substance, cell fractionation is the first step to be performed. There are several processes for cell fractionation, such as, enzymatic digestion, cell lysis, ultrasonic fragmentation, freeze-thaw, etc. However, these methods are mostly expensive and sometimes, different types of reagents may need to be added. Therefore, a low-cost and easier method is required for cell fractionation. We have developed a methodology by using ferrite particles, whose motion is controlled using an AC voltage source with low frequency. The system works like the ball mill method, where the ferrite particles act similar to ceramic balls. The motion of ferrite particles is controlled by the magnetic flux produced by an electromagnet aligned vertically to the treatment container. As ferrite particles are not toxic to biological substances, they are kept together under AC magnetic flux. The ferrite particles collide with cells and thus cell fractionation occurs, leading to protein extraction. We have measured the protein as well as nucleic acid concentration of the treated biological substances and observed increase in protein concentration after the treatment. Thus, our method can peovide a simple and easy technique for cell fractionation in the field of protein analysis.
\end{abstract}

Keywords: protein, cell fractionation, excess sludge, ferrite particles

\section{Introduction}

In several branches of scientific areas as well as daily life, the importance of protein extraction from bilogical substances is in great demand. Protein is being used in many ways such as production of medicine, detergent materials, food and also in genome analysis ${ }^{(1)-(3)}$. In order to extract protein from the biolgical substances, the cell fraction and protein purification are necessary (please see Fig. 1). Several methods are popular in cell fractionation process such as ultrsonic fragmentation, chemical treatment, freeze-thaw etc. ${ }^{(4)-(6)}$. These existing methods are good enough to fractionate biological cells but at the same time, they are very costly and not easy to operate.

Our research group had been working on biological cell lysis by controlling megnetic flux and using ferrite particles $^{(7)-(11)}$. Our previous works showed that the motion of ferrite particles controlled with an AC magnetic flux, could break up the cell wall or cell membrane and thus could lead to a reduction for excess activated sludge in water purification line of waste water treatment system ${ }^{(10)-(12)}$. Since this method deals with cell lysis, it can also be used as a cell fractionation

\footnotetext{
a) Correspondence to: Masaya Endo. E-mail: wister0317@gmail. com

* Department of Electrical and Electronic Engineering, Graduate School of Engineering and Resource Science, Akita University 1-1, Tegata Gakuenmachi, Akita-shi, Akita 010-8502, Japan

** Tohoku University of Community Service and Science 3-5-1, Iimoriyama Sakata-shi, Yamagata 998-8580, Japan
}

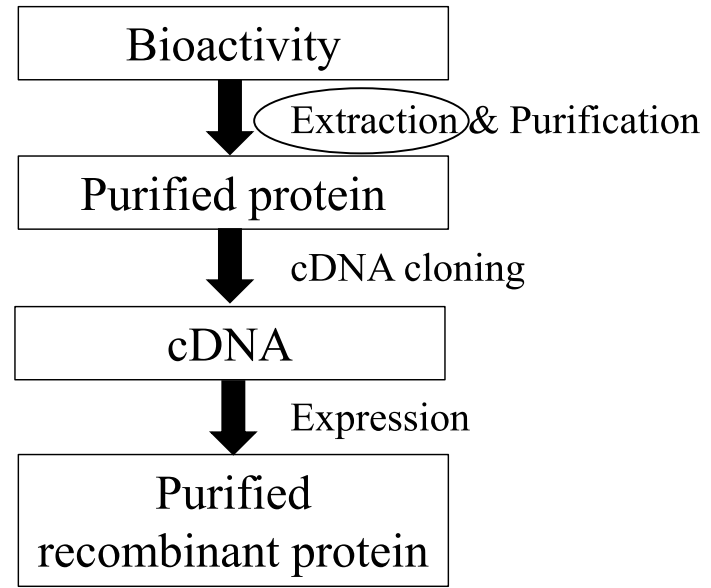

Fig. 1. Protein analysis ${ }^{(4)}$

process with proper investigation.

The idea to fractionate cell is similar to the reduction process of excess activated sludge. But the system and experiment's condition needs more cautiousness for extracting protein from biological cells. We have developed a new method for cell fractionation which uses ferrite particles and $\mathrm{AC}$ magnetic flux. The movements of ferrite particles are controlled by the AC magnetic flux like the previous report ${ }^{(12)}$. At first, the biological materials was kept with a certain amount of ferrite particles. When the magnetic flux is applied, the movement of ferrite particles will crush the cell wall or cell membrane of the biological cells. Thus, the protein can be 
extracted from the cells. Ferrite particles are non-toxic and safe for biological substances ${ }^{(13)}$. They can be easily separated from sludge by using magnets. This method is less expensive comparing to other methods. We have achieved good results in extracting protein from the cells by using our method.

\section{Experimental Method}

In this research work, we used activated sludge as the biological substances. Activated sludge is a group of biological organisms which can clean water by removing biological waste from waste water. Activated sludge is used in waste water treatment plants for purifying waste water and sewerage treatment. The seed sludge for this research work was collected from Akita Sewerage Treatment Plant of Akita city, Japan and cultivated at laboratory scale in Akita University.

The experimental setup and the circuit model can be seen in Fig. 2. A coil (self inductance: $7.8 \mathrm{H}$ ) was set up in vertical direction and a treatment container was placed beneath it. The coil was connected with an AC voltage source (BP4610, NF). The coil was connected with 2 diodes (GSF05A40, VRRM $=400 \mathrm{~V}$, IFAV $=5 \mathrm{~A}$ ) which were installed in opposite direction to each other. The diodes were set up with the coil (EM), and when the coil (EM) was connected with AC power supply, the electric current was provided alternative directions to the coil. Thus, the coil become electromagnet with a gap of time period with the AC voltage source with a certain frequency. A certain amount of ferrite particles and activated sludge were poured into the treatment container. Ferrite particles are magnetic substances and they can be moved easily with necessary magnetic flux density. While they moved in the container, collisions might occur with the cells of activated sludge. For a certain AC power supply with frequency, these collisions may break the cell wall or cell membrane of bioorganisms of activated sludge. Thus, cell lysis was achieved and protein was extracted.

The movement of ferrite particles can be controlled by the electromagnet with AC voltage application. The electrical and magnetical properties are known to provide the ferrite particles' motion. At first, we measured the $I-V$ relationship with square wave. Due to the limit of the voltage source,

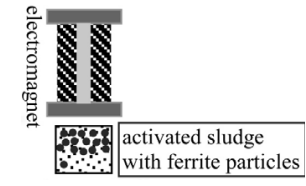

(a) electromagnet setup

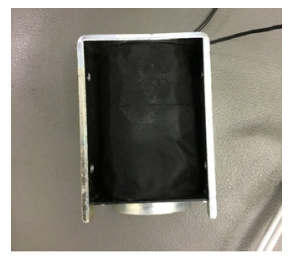

(c) electromagnet

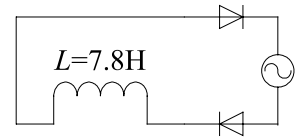

(b) circuit diagram

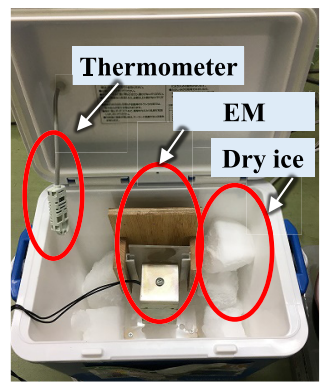

(d) treatment setup
Fig. 2. Experimental setup the applied voltage was in the range of $0-120 \mathrm{Vp}-\mathrm{p}$. The electromagnetical characteristics of the coils were measured by a Gauss meter (GM04, HIRST MAGNETIC Instrument). The size of the ferrite particles is an important factor for this method. We chose the shape to be round and size to be less than $53 \mu \mathrm{m}$ in this experiment on the basis of previous works $^{(7)-(12)}$. Then, we determined the size and the shape of the treatment container. The amount of the activated sludge is dependent on the size of the container. And the size and shape of the container depend on the magnetic flux density distribution produced by the coil (EM). The measurement process for magnetic flux density will shortly be described here. The Hall probe of the gauss meter was set under the core of the coil. The center of the core of coil was chosen as the center of the coordinates. The magnetic flux density was measured from -2 to $2 \mathrm{~cm}$ in the directions along with $\mathrm{X}$ and $\mathrm{Y}$ axes. The DC peak mode was selected for the measurement. The voltage was applied by the bipolar voltage source. The magnetic flux density values were measured for $50 \mathrm{Vp}$-p to $120 \mathrm{Vp}$-p by increasing $10 \mathrm{Vp}$-p for input voltages. The magnetic flux density distribution was calculated for each input voltage. Thus, after evaluating the electrical and magnetical properties, we utilized them for several measurements related to the protein extraction of activated sludge.

The movement of ferrite particles is possible only inside the magnetic flux produced by the coil (EM). So, the size and shape of the treatment pot is related to the magnetic flux density distribution of the coil (EM). We measured the magnetic flux density on the $\mathrm{Z}$ direction (vertical direction) also.

These data were helpful to decide the size and shape of the treatment container. The ferrite particles and activated sludge were poured into this container and kept under the core of the coil. The treatment was performed for a certain period of time and for different concentrations of activated sludge. Activated sludge was taken from the aeration tank. The MLSS (Mixed Liquor Suspended Solids) was measured by an electronic moisture meter (120H MOC, Shimadzu Co., Japan). The values of MLSS were adjusted if needed. A certain amount of activated sludge was taken to the container with $2.3 \mathrm{~g}$ of ferrite particles. The amount of ferrite particles were decided referring to the previous papers ${ }^{(7)-(12)}$ published in other journals. The whole setup of the system was put in a heat insulated box where a certain amount of dry ice was kept in order to keep the system in lower temperature (Fig. 2(d)). For different experiment time, the same amount of activated sludge was kept at a refrigerator which temperature was controlled at $5^{\circ} \mathrm{C}$ without any treatment. Again, a thermometer was set up inside the thermo insulated box to monitor the temperature of the experimental environment.

After the treatment to the activated sludge, the temperature of the liquid of treatment container was measured by a thermometer (450WR CT, CUSTOM Co., Japan). Then the protein extraction amount of the activated sludge was investigated. The treated activated sludge was separated from ferrite particles by a parmanent magnet. Then the separated liquid was put in a glass test tube and then centrifuged by a centrifugal separator (607 HC, Central Kagaku Co., Japan). The supernatant of the centrifuged liquid were collected and the protein concentration and the nucleic acid concentration were measured by a spectro UV meter (Nanodrop 2000c, Thermo 


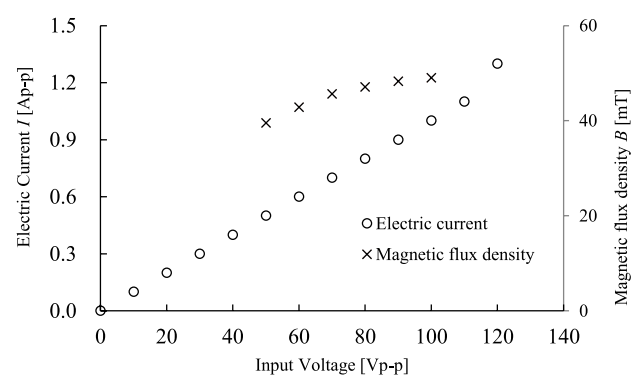

Fig. 3. I-V and B-Vcharacteristics of the electromagnet (EM)

Scientific Co., USA) with 2 different wavelengths of UV ray.

\section{Results and Discussions}

3.1 I- $V$ Realationship The $I-V$ relationship of the coil (EM) and input voltage was determined. The r.m.s. value was calculated for both voltage and electric current for the coil (EM). The electrical characteristics were measured for square wave only. The frequency of the voltage source was fixed at $1.0 \mathrm{~Hz}$. Figure 3 shows the measured data of current $I$ and magnetic flux density $B$ with different values of input voltages. The electric current $I$ increased almost linearly in the coil (EM) with input voltage. Since the magnetic flux density $B$ is proportional to the electric current $I$, so it can be understood that the magnetic flux density $B$ also increased with current. For the maximum range of input voltage (120 Vp-p), the maximum value of current was found at 1.3 Ap-p in a coil. However the magnetic flux density was measured only upto $100 \mathrm{Vp}-\mathrm{p}$ as the upper voltage limit of the coil was $100 \mathrm{Vp}$-p. The magnetic flux density was found increasing with input voltage linearly, but around $80 \mathrm{Vp}-\mathrm{p}$, it started to become saturated. This magnetic flux density was sufficient enough to move ferrite particles ups and downs in a treatment container in this experiment.

The values of electric current and the magnetic flux density may be influenced by temperature rise in coil and the frequency of input voltage. From the view of electric circuit, the impedance of the coil depends on the frequency and temperature as they are related with the imepedance of the coil i.e. $\omega L$ and resistance $R$. Here, $\omega[\mathrm{rad} / \mathrm{s}]$ is the angular frequency, $L[\mathrm{H}]$ is the self inductance and $R[\Omega]$ is the resistance of the coil. The resistance $R$ rises with temperature of the coil (EM). And an electromagnet (EM) can be overheated due to long time electric current flow in it and which may decrease the magnetic flux during operating period. As we put all the setting in a thermo insulated box with dry ice, the temperature was found around $0^{\circ} \mathrm{C}$ althrough the experiment period. Thus the effect of temperature rise was avoided in this experiment system. Again, the impedance effect for the frequency was also to be considered. If the frequency of the voltage source is doubled then the value of impedance becomes double comparing to that of initial value and it decreases the value of current as well as magnetic flux. The frequency controls the movement of the ferrite particles but at the same time, if it increases then the impedance will also increase. And the electric current $I$ and the magnetic flux density $B$ will be decreased. So, it can be understood that ferrite particles cannot move well with larger frequency.

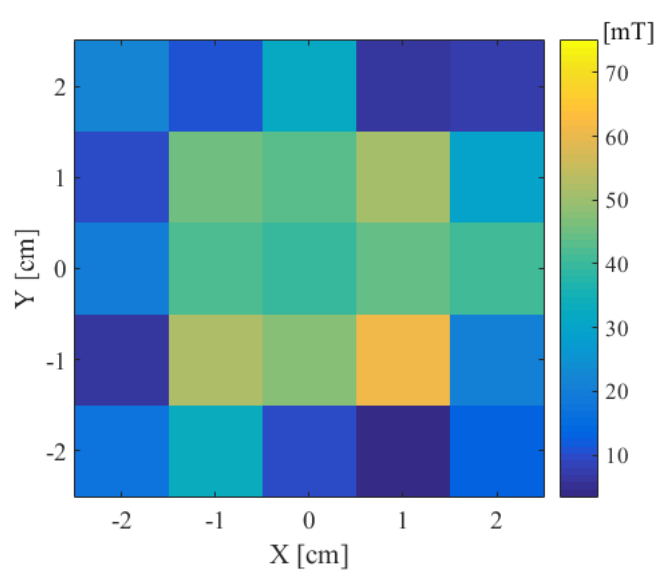

(a) for input voltage of $50 \mathrm{Vp}-\mathrm{p}$

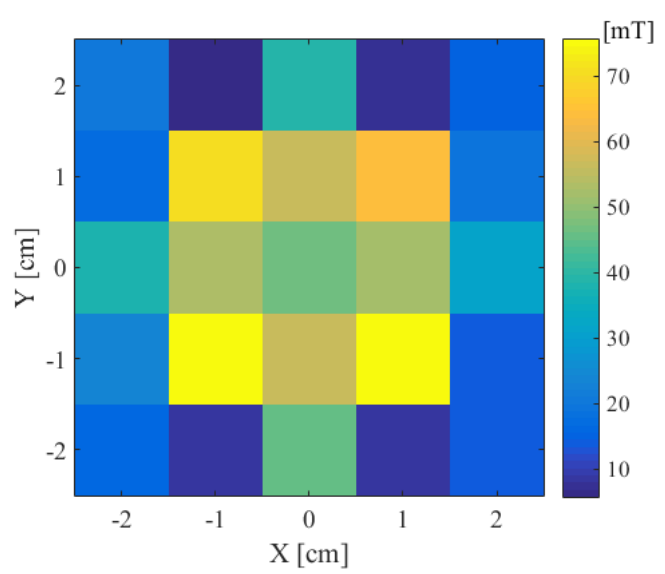

(b) for input voltage of $80 \mathrm{Vp}-\mathrm{p}$

Fig. 4. Magnetic flux density distribution for the electromagnet (EM) with different input voltages

3.2 Treatment Container The shape and the size of the treatment container was determined with magnetic flux density distribution for the electromagnet (EM). We measured the magnetic flux density of the coil by using the gauss meter with input voltage $50 \mathrm{Vp}$-p to $100 \mathrm{Vp}$-p by increasing $10 \mathrm{Vp}$-p. Figure 4 shows the magnetic flux density distributions of the coil for $50 \mathrm{Vp}-\mathrm{p}$ and $80 \mathrm{Vp}$-p of input voltages. We can see the magnetic flux density distribution with square wave was quite uniform around the core of the coil (EM). The values of the magnetic flux density $B$ was found larger for larger input voltage. In this experiment, we chose $80 \mathrm{Vp}$ $\mathrm{p}$ as the input voltage. The magnetic flux density produced by the coil (EM) with applied voltage and frequency at the vertical direction was also measured. The diameter of the container was chosen with the diameter of core of the electromagnet (i.e. $1.5 \mathrm{~cm}$ ). The data are not shown here but the magnetic flux density became weaker at $2 \mathrm{~cm}$ height from the ground. So the height of the container was chosen at $2 \mathrm{~cm}$. The capacity of the container was $10 \mathrm{~mL}$. The material of the container was soft polyethelene and the shape was cylindrical $(\phi 15 \mathrm{~mm} \times 61.5 \mathrm{~mm})$. Considering the previous results of magneto-ferrite treatment, $2.3 \mathrm{~g}$ of ferrite particles were taken into the container with $10 \mathrm{~mL}$ of activated sludge ${ }^{(7)(10)(12)}$. The treatment was applied for different time and different MLSS concentrations of activated sludge.

3.3 Temperature Rise The treatment was performed with the determined parameters. The treatment container 
Table 1. Temperature measurement during treatment time

\begin{tabular}{c|c|c}
\hline \multirow{2}{*}{ time $[\mathrm{min}]$} & \multicolumn{2}{|c}{ Temperature $\left[{ }^{\circ} \mathrm{C}\right]$} \\
& control & after treatment \\
\hline 5 & & \\
\hline 10 & \multirow{3}{*}{4} & 4 \\
\hline 15 & 4 & \\
\hline 30 & & 10 \\
\hline $6 n$ & & 18.4 \\
\hline
\end{tabular}

with $10 \mathrm{~mL}$ of activated sludge and $2.3 \mathrm{~g}$ of ferrite particles was set up under the coil (EM). The ferrite particles in the treatment pot can come down from the upper side of the treatment pot due to the gravitational force working on positive direction on them. But, the gravitational force turns into opposite direction to the ferrite particles when they move upwords due to the magnetic flux applied on them. At first the temperature rise was considered with MLSS of $3000 \mathrm{mg} / \mathrm{L}$ of activated sludge. The measured data is shown in Table 1 . The activated sludge was treated under different values of treatment time and after each treatment, the container cap was open for the temperature measurement. The temperature was measured by a thermometer. No temperature rise was found upto 15 min of treatment time comparing to the control setup. But the temperature of the treated activated sludge was increased significantly after $30 \mathrm{~min}$. of treatmernt. The amount of protein can be effected by temperature rise ${ }^{(4)}$ and we chose treatment time upto $15 \mathrm{~min}$ for further experiments.

3.4 Protein Extraction Three values of MLSS were chosen (i.e. $3000,5000,8000 \mathrm{mg} / \mathrm{L}$ ) to observe the extracted protein amount and nucleic acid concentration with this method. After the treatment, the activated sludge was taken to a test tube for centrifuging with $3000 \mathrm{rpm}$ for $10 \mathrm{~min}$. Then, the supernatant liquid from each sample including the control sample were measured by Nanodrop 2000c. The protein concentration of the treated sample was measured at $280 \mathrm{~nm}$ of UV ray and the nucleic acid concentration was measured at $340 \mathrm{~nm}$ of UV ray at the same time. Thus the amount of nucleic acid concentration and protein concentration were measured for each sample. Each sample was measured for three times and then their average values were determined. The value of standard deviation for each sample were also calculated. The results can be seen in Fig. 5 . Both the values of protein and nucleic acid concentration were larger than that of control sample. However, the protein amount was found smaller comparing to control sample for $5000 \mathrm{mg} / \mathrm{L}$ of activated sludge sample. We performed the same experiment for $5 \mathrm{~min}$ with $5000 \mathrm{mg} / \mathrm{L}$ of activated sludge and the result was found different comparing to that of Fig. $5(0.14 \mathrm{mg} / \mathrm{mL}$ for protein and $10.0 \mu \mathrm{g} / \mu \mathrm{L})$. The values of new experiment's results were even larger than the $8000 \mathrm{mg} / \mathrm{L}$ of activated sludge's experiment. It is obvious that the amount of protein and nucleic acid concentration from activated sludge does not depend only on the the electrical properties of the electromagnet (EM) but it also depends on the the activated sludge itself. These experiments were performed in different days and it was quite difficult to recognize the biological condition of the activated sludge when we

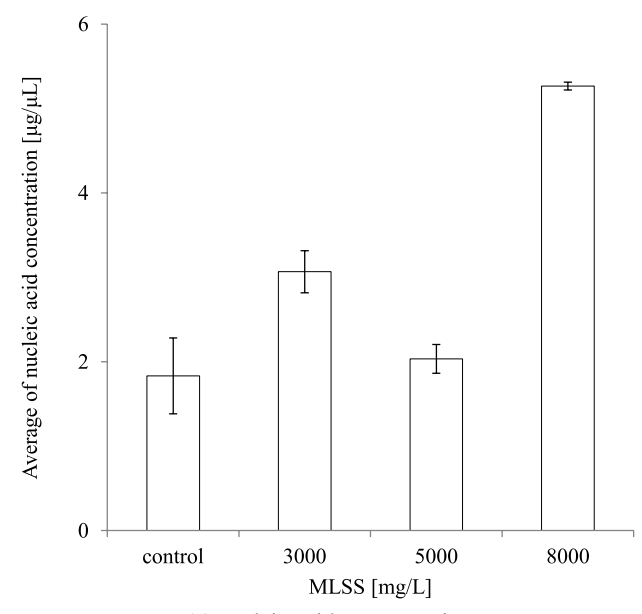

(a) nucleic acid concentration

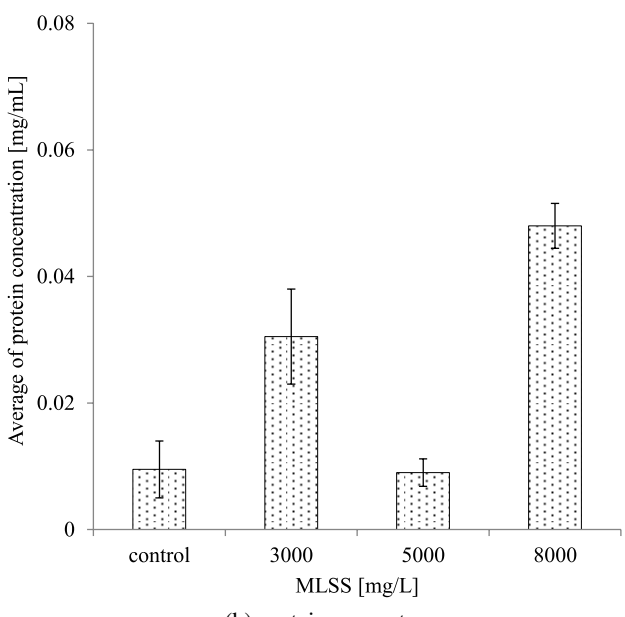

(b) protein amount

Fig. 5. Protein amount and nucleic acid concentration due to cell fractionation with different MLSS

had performed the experiments. The activated sludge for this research was nurtured in a miniature waste water treatment plant in Akita University. There might be some problems in the activated sludge during that experiment time. However, for other samples of different MLSS showed the increase of nucleic acid concentration and protein with the MLSS values of the treatment activated sludge. Generally, it can be understood that for larger MLSS of activated sludge, the amount of protein and nucleic acid will be larger in amount as the MLSS indicates the larger amount of activated sludge and biological substances.

Next, we performed the same experiment for activated sludge with different treatment time. The MLSS of the activated sludge was chosen $3000 \mathrm{mg} / \mathrm{L}$ for all different treatment time experiments. 5 different experiment times were chosen (i.e. 1, 3, 5, 10 and $15 \mathrm{~min}$ ). Different samples with different time was chosen though their MLSS values were adjusted at $3000 \mathrm{mg} / \mathrm{L}$ before the experiment. Each sample was taken under the same process in order to measure their protein and nucleic acid concentration values. Again, they were measured for three times and after that, the average values were determined. The results can be seen in Fig. 6. It can be seen that the extracted protein and nucleic acid values are remarkable even from 3 min of treatment time. The amount of nucleic acid concentration increased gradually with time but 


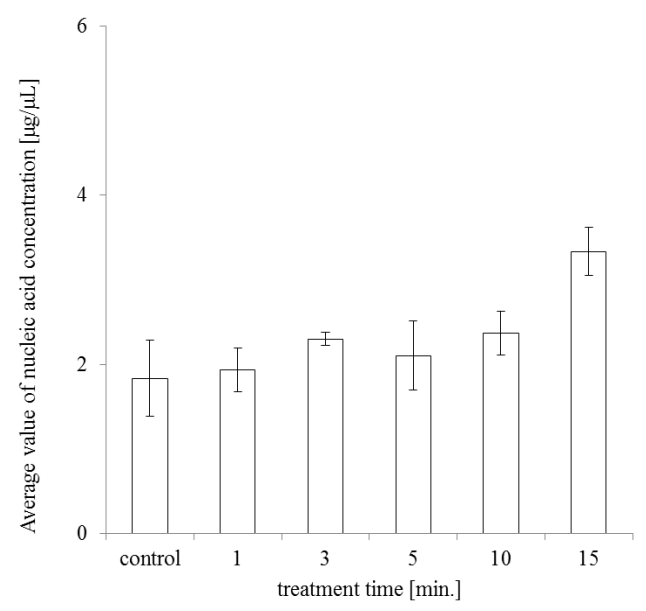

(a) nucleic acid concentration

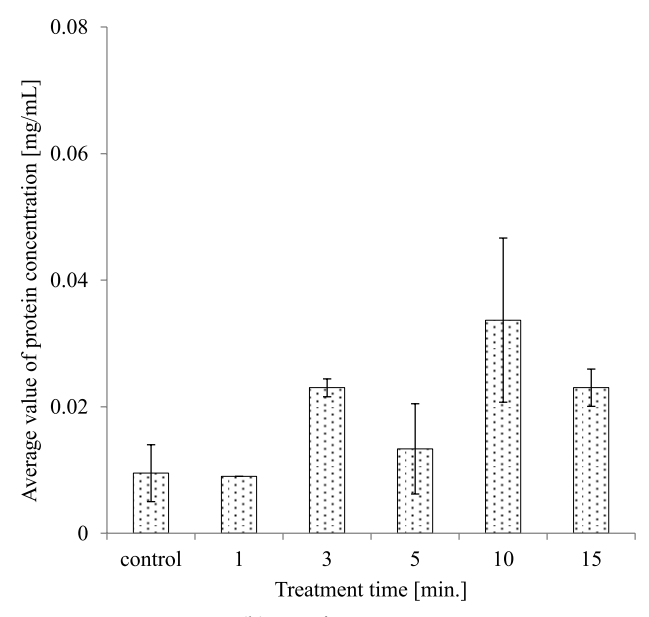

(b) protein amount

Fig. 6. Protein amount and nucleic acid concentration due to cell fractionation with different treatment time

for protein the amount showed increase for $3 \mathrm{~min}$ and $10 \mathrm{~min}$ of treatment significantly.

In our experiment, small amount (i.e. $2.3 \mathrm{~g}$ ) of ferrite particles were used. Ferrite particles are non toxic for biological substances $^{(13)}$ and they are very cheap. The market price of the ferrite particles is $¥ 1,500 / \mathrm{kg}$ (less than $\$ 15 / \mathrm{kg}$ ) in Japan. Another thing to be noted that the ferrite particles can be used several times as they were found without any damage after performing the experiments. The electric power consumption was also calculated for our experiments. The value of the total power consumption was only $0.16 \mathrm{kWh}$. Again, the voltage source and the coil (EM) are also not very costly comparing to the existing methods of cell fractionation.

Considering the results from our experiments and the cheaper costs of this method, no doubt to say that it can be used as a new and effective method for cell fractionation as well as extraction of protein. It can be applied for the cell fractionation in protein analysis.

\section{Conclusions}

In this research work, we have introduced a new method for cell fractionation which was confirmed by measuring the protein and nucleic acid concentration measurement after the treatment of activated sludge. This method for cell fractionation is easier and cheaper comparing to other cell fractionation methods. Thus protein extraction from bilogical substances will pave the way of using the biological industrial waste such as activated sludge for protein extraction also. More research work will be needed for different types of biological substances for cell fractionation experiments with this new method.

\section{References}

( 1 ) J. Cui, A. Dong, W. Zhang, and F. Miao: "Experimental investigation of extracting protein by alkaline thermal sludhe hydrolysis from sludge", Chin. J. Environ. Eng., Vol.3, No.10, pp.1889-1892 (2009)

( 2 ) G. Bindea, B. Mlecnik, M. Tosolini, A. Kirilovsky, M. Waldner, A. Obenauf, H. Angell, T. Fredriksen, L. Lafontaine, A. Berger, P. Bruneval, W. Fridman, C. Becker, F. Pagès, M. Speicher, Z. Trajanoski, and J. Galon: "Spatiotemporal dynamics of intratumoral immune cells reveal the immune landscape in human cancer", Immunity, Vol.39, No.4, pp.782-795 (2013)

( 3 ) L. Aegliani, J. O'Regan, A.L. Kelly, and J.A. O'Mahony: "Composition and protein profile analysis of rice protein ingredients", Journal of Food Composition and Analysis, Vol.59, pp.18-26 (2017)

( 4 ) Experimental Notes on Protein Experiment (1st volume): Ed. M. Okada and H. Miki, Yodosha, Tokyo (2015)

( 5 ) R. Zhang, J. Chen, and X. Zhang: "Extraction of intracellular protein from ethanol soaking, enzyme digest, ultrasonication and homogenization techniques”, Bioresource Technology, Vol.247, pp.267-272 (2018)

( 6 ) H.K. Kim, Y.H. Kim, Y.E. Kim, S.K. Jung, N.H. Lee, and K.-M. Song: "Effects of salts on ultrasonic extraction of protein from porcine myocardium", Food and Bioproducts Processing, Vol.108, pp.12-17 (2018)

( 7 ) M. Kabir, M. Suzuki, and N. Yoshimura: "Reduction of Excess Sludge by Ferrite Particles", Jpn. J. Wat. Treat. Biol., Vol.43, No.4, pp.189-197 (2007)

( 8 ) M. Kabir, M. Suzuki, and N. Yoshimura: "Reduction of Excess Sludge by Magneto-Ferrite Treatment: Observation on Lab Scale WWTPs", IEEJ Trans. Elec. Electron. Engg., Vol.4, No.4, pp.584-586 (2009)

( 9 ) M. Kabir, M. Suzuki, and N. Yoshimura: "Reduction of Excess Activated Sludge by Ferrite Particles: Methods for Practical Use", Int. J. Soc. Mater. Eng. Resour, Vol.17, No.2, pp.120-125 (2010)

(10) M. Kabir, M. Suzuki, and N. Yoshimura: "Excess activated sludge reduction by using electromagnets and frrite particles", IEEJ Trans. Elec. Electron. Engg., Vol.7, No.2, pp.220-224 (2012)

(11) M. Kabir, M. Endo, M. Suzuki, and N. Yoshimura: "Study on the Treatment of Excess Activaed sludge on the Return Sludge Line", IEEJ Trans. Indust. Appl., Vol.134, No.2, pp.239-240 (2013) (in Japanese)

(12) M. Endo, M. Kabir, M. Suzuki, and N. Yoshimura: "Reduction of Excess Sludge by Magnetoferrite Treatment Applied to the Return Sludge", IEEJ Trans. Indust. Appl., Vol.136, No.3, pp.198-203 (2016) (in Japanese)

(13) B. Issa, I.M. Obaidat, B.A. Albiss, and Y. Haik: "Magnetic Nanoparticles: Surface Effects and Properties Related to Biomedicine Application", Int. J. Mol. Sci., Vol.14, pp.21266-21305 (2013)

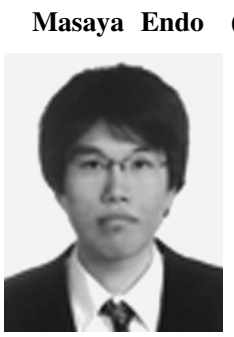

(Member) received the B.E. and M.E. degrees in Electric Engineering from Akita Univercity,Akita Japan,in 2013 and 2015 respectively. He is currently studying in the doctoral course from October 2015. $\mathrm{He}$ is a Member of the IEE of Japan. He is working on reduction of waste activated sludge by using Ferrite Particles'Motion Controlling System. 
Mahmudul Kabir (Member) is an associate professor at Graduate

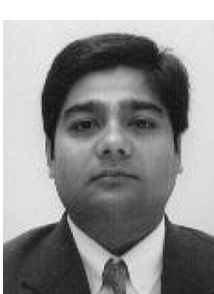
School of Engineering Science, Akita University, Japan. He completed his High School education in Bangladesh. He received his B.E., M.E. and Ph.D. degrees in Electrical and Electronic Engineering from Akita University in 2000, 2002 and 2005 respectively. $\mathrm{He}$ is a member of the IEE of Japan, the Institute of Electrostatics Japan, IEEE and Japanese Society of Water Treatment Biology.
Noboru Yoshimura (Life Member) received his B.E. and M.E. from

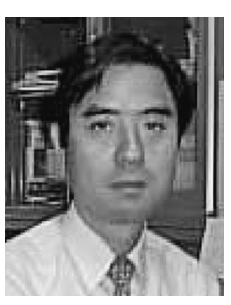
Akita University in 1967 and 1969, respectively and Dr. degree from Nagoya University in 1975 . He joined Akita University in 1969. He became a professor in 1983 and the Dean of Mining College of Akita University in 1995. Now he is the President of Tohoku University of Community Service and Science since since 2014. He also worked in Clarkson University of USA as a visiting scholar from 1978 to 1979 and as a visiting professor in 1989 . He received paper award from IEE of Japan in 1984. He is now advisory professor of Xi' an Jiaotong University in China and had been president of The Society of Material Engineering for Resource of Japan until 1999. His research interests include organic dielectric materials, dielectric and semi-conductive ceramics. He is a member of IEEE, The Illuminating engineering of Institute of Japan and other several Institutes and Societies. 